\title{
Dosimetric Advantages of Volumetric Modulated Arc Therapy Based Coronal Arc Delivery Technique in Brain Stereotactic Radiosurgery: A Feasibility Study
}

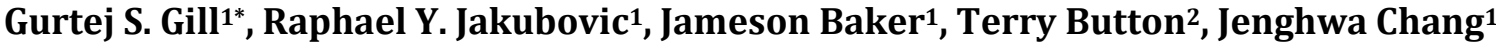 \\ ${ }^{1}$ Department of Radiation Medicine, Center for Advanced Medicine, Northwell Health, Lake Success, NY, USA \\ ${ }^{2}$ Department of Radiology and Biomedical Engineering, Stony Brook University, Stony Brook, NY, USA \\ Email: *ggill1@northwell.edu
}

How to cite this paper: Gill, G.S., Jakubovic, R.Y., Baker, J., Button, T. and Chang, J. (2019) Dosimetric Advantages of Volumetric Modulated Arc Therapy Based Coronal Arc Delivery Technique in Brain Stereotactic Radiosurgery: A Feasibility Study. International Journal of Medical Physics, Clinical Engineering and Radiation Oncology, 8, 80-94.

https://doi.org/10.4236/ijmpcero.2019.82008

Received: February 12, 2019

Accepted: March 31, 2019

Published: April 3, 2019

Copyright $\odot 2019$ by author(s) and Scientific Research Publishing Inc. This work is licensed under the Creative Commons Attribution International License (CC BY 4.0).

http://creativecommons.org/licenses/by/4.0/

\section{Abstract}

The feasibility of a volumetric modulated arc therapy (VMAT) based coronal arc (cARC) technique for treating a single brain metastasis or lesion proximal to the brainstem or optic chiasm was evaluated. Coplanar (CP) and non-coplanar (NCP) treatment plans to an anthropomorphic head/neck phantom scanned head-first supine were compared to a cARC plan with the phantom rotated vertically. A set of planning target volumes (PTVs) were contoured centrally between the brainstem and optic chiasm ("Ant PTVs") and posterior to brainstem ("Post PTVs"). Dosimetric indices such as conformity index (C.I.), gradient measure (G.M.), and dose volume histograms (DVHs) were compared for CP, NCP and cARC techniques. The TG101 guidelines for organs-at-risk (OARs), and $95 \%$ of PTV receiving at least $100 \%$ of the prescription dose (D95 $=100 \%)$ were used as plan objectives. Reductions in D50 and D30 to the brainstem of $85.1 \% \pm 3.9 \%$ and $87.6 \% \pm 3.2 \%$, respectively were seen for "Post PTVs", and $51.1 \% \pm 17.8 \%$ and $85.6 \% \pm 6.0 \%$ respectively for "Ant PTVs" using cARC versus CP ( $\mathrm{p} \leq 0.01)$. For chiasm, reductions of D50 and D30 were $61.7 \% \pm 3.2 \%$ and $44.2 \% \pm 8.9 \%$ for "Ant PTVs", by $69.3 \% \pm$ $8.0 \%$ and $74.3 \% \pm 8.2 \%$ for "Post PTVs" ( $\mathrm{p} \leq 0.01$ ). Comparing cARC to NCP led to similar dosimetric improvements. The conformity index (C.I.) was measured to be $1.101 \pm 0.038,1.088 \pm 0.054$, and $1.060 \pm 0.040$ for cARC, CP and NCP respectively $(\mathrm{p} \leq 0.01)$. The overall GM in $\mathrm{cm}$ was $0.581 \pm 0.097$, $0.708 \pm 0.064$, and $0.476 \pm 0.050$ for cARC, CP and NCP respectively ( $\mathrm{p} \leq$ 0.01 ). The mean distance gradient fall-off (in $\mathrm{cm}$ ) was $0.249 \pm 0.038$ (cARC), $0.749 \pm 0.107(\mathrm{CP})$, and $0.621 \pm 0.068(\mathrm{NCP})$ at the center slice in anterior-posterior direction of the target volume $(\mathrm{p} \leq 0.01)$. The objective of this 
study is to compare the dosimetric indices of cARC with CP and NCP techniques. In conclusion, cARC can provide improved dosimetry as compared to $\mathrm{CP}$ and NCP for lesion proximal to the brainstem or optic chiasm.

\section{Keywords}

VMAT, Coronal Arc, $\pi$-Geometry, Coplanar, Non-Coplanar, Stereotactic Radiosurgery, Coronal Arc

\section{Introduction}

The evolution of treatment planning, specific to patient treatment design and delivery has had a profound clinical impact, facilitating improved treatment outcomes while sparing the organs-at-risk (OARs) [1] [2] [3] [4] [5]. This development, with respect to brain pathologies, has enabled treatment of targets adjacent or near critical structures including the eyes, lenses, optic nerves, optic chiasm, brainstem and cochlea. These OARs pose significant challenges to the medical physicists/dosimetrists, responsible for safe and accurate treatment delivery. Intensity modulated radiation therapy (IMRT) [6] represents a major improvement over traditional forward planning techniques by utilizing computerized inverse planning and modulating the intensity of the radiation beam based on specific planning criteria including OARs dose constraints or PTV coverage goals. These advantages have become increasingly pronounced with the introduction of coplanar (CP) and non-coplanar (NCP) volumetric modulated arc therapy (VMAT) [7]. In these applications dose modulation is performed by optimizing gantry rotation, dynamic jaw/multileaf collimator (MLC) motion, and dose rate to achieve efficient dose delivery and superior OAR sparing.

Coronal arc (cARC) treatment planning consists of VMAT delivery in a coronal plane of the brain $\mathrm{CT}$ and differs from the axial plane delivery specifically with regard to beam orientation and delivery. In this study we evaluated the potential of VMAT based cARC technique. Since currently available treatment planning systems do not allow for patient rotation during beam delivery, the cARC technique was simulated using a vertically scanned anthropomorphic head and neck phantom. Each simulated cARC plan consisted of two multi-segmented partial $\left(270^{\circ}\right.$ to $0^{\circ}$ and $0^{\circ}$ to $90^{\circ}$ gantry angles) VMAT arcs, with dose modulation delivered along the coronal plane of phantom. Here we compare dosimetry results for the cARC to conventional coplanar and non-coplanar treatment of lesions of the brainstem or optic chiasm. Ongoing advancements in VMAT planning have the potential to provide crucial dose sparing of intracranial OARs, especially during hypo fractionated stereotactic radio-surgery (SRS) regimens.

\section{Materials and Methods}

\subsection{Treatment Planning Study}

The head and neck Alderson Rando anthropomorphic phantom (Model\# ART 
210: RSD Alderson Phantoms, Long Beach, California) was scanned axially using our in-house CT-SIM (Siemens Medical Solutions USA, Inc.) both horizontally and vertically $(120 \mathrm{KVp}, 200 \mathrm{mAs}, 515 \times 515$ matrix size, $50 \mathrm{~cm}$ field of view, and $2 \mathrm{~mm}$ slice thickness) and exported to the Eclipse Treatment Planning system (Version \#15.6, Varian Medical Systems, Palo Alto, CA) for treatment planning. PTVs were selected to simulate conditions where the cARC technique might be particularly advantageous as indicated in Figure 1. The PTVs were contoured posterior to the brainstem (Post PTVs) and centrally between the brainstem and optic chiasm (Ant PTVs). The set of "Ant PTVs" and "Post PTVs" were drawn to evaluate the effectiveness of cARC delivery. Varying distances from OARs (i.e. brainstem and/or optic chiasm) were used for each set of PTVs to assess the potential benefits of the cARC technique. The naming convention and descriptions for all PTVs are provided in Table 1.

Treatment planning consisted of a stereotactic radiosurgery (SRS) approach delivering $21 \mathrm{~Gy}$ in a single fraction using $6 \mathrm{MV}$ flattening free filter (FFF) photon beams. Treatment planning was performed using a high-definition multileaf collimator (HD-MLC) system consisting of 120 total leave (32 central $2.5 \mathrm{~mm}$ width pairs +28 peripheral $5 \mathrm{~mm}$ width pairs) and $1.5 \mathrm{~mm}$ dose grid resolution for all treatment plans. In the current study we consider two conventional treatment design approaches-standard coplanar (CP) arcs and non-coplanar (NCP) arcs were compared to the novel cARC approach as shown in Figure 2.

\subsection{Coplanar Arcs (CP)}

The coplanar VMAT technique consists of multiple arcs planned in a single axial plane to facilitate the delivery of higher doses in regions of beam intersection. The Varian RapidArc ${ }^{\circledast}$ [8] approach consists of variable dose rate, variable gantry speed and dynamic MLCs. For the purpose of the study two coplanar arcs rotating clockwise from $181^{\circ}-179^{\circ}$ and counter-clockwise from $179^{\circ}$ to $181^{\circ}$ were utilized with a stationary couch angle of $0^{\circ}$.

\subsection{Non-Coplanar Arcs (NCP)}

The non-coplanar arc technique utilizes multiple beam geometries strategically planned using non-standard couch angles. This technique is advantageous in the context of radiosurgery, where the goal of treatment is to deliver an ablative tumorcidal dose to the lesion while sparing the adjacent critical structures. It offers significant advantage over coplanar arcs when considering the dose delivered to the target by looking at the conformity of high isodose lines such as $80 \%-95 \%$. Therefore, non-coplanar arc therapy has the ability to shift the distribution away from the critical structures because the dose distribution follows the beam entrance. Use of non-standard couch angles can impose concerns of collision but with careful selection of couch and gantry angle collisions can be avoided. For this study three arcs were utilized clockwise: gantry angle $0^{\circ}-179^{\circ}$ at couch angle $45^{\circ}$; anticlockwise: gantry angle $0^{\circ}-181^{\circ}$ at couch angle $315^{\circ}$ along with one coplanar arc mentioned earlier in the "coplanar arcs" section. 


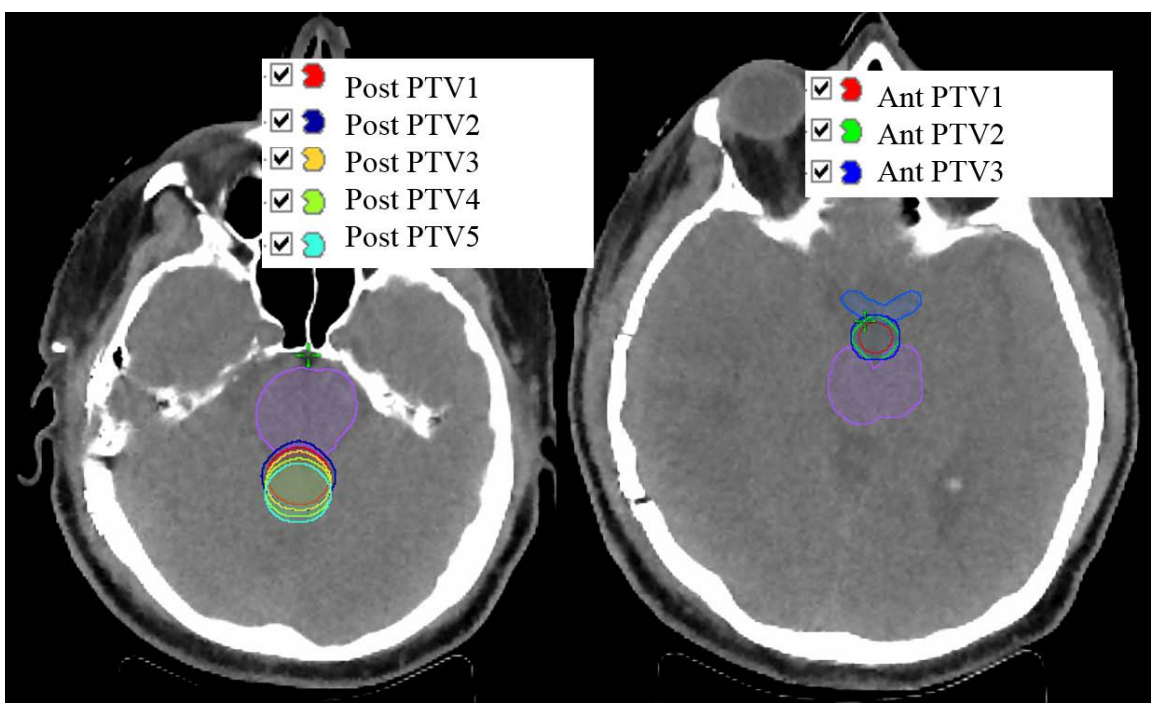

Figure 1. Simulated planning target volumes with incremental margins near organs at risk. "Post PTVs" (Left Panel) is located posterior to the brainstem. "Ant PTVs" (Right Panel) is located in between the optic chiasm and the brainstem.

\begin{tabular}{|c|c|c|}
\hline $\begin{array}{c}\text { Coplanar ARC technique, } \\
2 \text { full ARCs }\end{array}$ & $\begin{array}{c}\text { Non-coplanar ARC technique, 1 } \\
\text { full ARC and } 2 \text { half ARCs }\end{array}$ & $\begin{array}{c}\text { Coronal ARC tecnique, } 2 \text { half } \\
\text { ARCs (combination of both } \\
\text { ARCs represents a } \pi \text {-geometry) }\end{array}$ \\
\hline & &
\end{tabular}

Figure 2. Beam orientation for coplanar (CP), non-coplanar (NCP) and coronal arc (cARC) techniques.

Table 1. SRS Planning Treatment Volumes (PTVs) with associated description of margins.

\begin{tabular}{cc}
\hline SRS Planning Treatment Volumes (PTVs) & Description of margins \\
\hline Ant PTV1 & PTV itself \\
Ant PTV2 & $1 \mathrm{~mm}$ margin from “antPTV1" \\
Ant PTV3 & $2 \mathrm{~mm}$ margin from "antPTV1" \\
Post PTV1 & PTV itself \\
Post PTV2 & $1 \mathrm{~mm}$ margin from “postPTV1" \\
Post PTV3 & $2 \mathrm{~mm}$ posterior from "brainstem" \\
Post PTV4 & $4 \mathrm{~mm}$ posterior from "brainstem" \\
Post PTV5 & $6 \mathrm{~mm}$ posterior from "brainstem” \\
\hline
\end{tabular}

\subsection{Coronal Arc (cARC) Delivery Technique}

The cARC technique utilizes two partial arcs delivered in a coronal plane. The main difference of cARC from standard VMAT delivery is delivering dose in a 
coronal plane by rotating the couch while the gantry is fixed at $90^{\circ}$ or $270^{\circ}$. Since our treatment planning system doesn't allow couch rotation during the beam delivery, we simulated the cARC technique in this study by scanning the head and neck anthropomorphic phantom in a vertical position. The cARC beams were simulated with two VMAT beam at gantry angles of $0^{\circ}-90^{\circ}$ and $270^{\circ}-0^{\circ}$ respectively, with couch angle $0^{\circ}$. These partial arcs represent a $\pi$-geometry. The treatment planning design for this approach is demonstrated in Figure 2.

\subsection{SRS Planning Criteria}

Dosimetric indices for CP and NCP techniques were compared with the CARC technique. During the optimization process, the dose constraints to PTVs and OARs were kept constant for all plans. In addition, all plans were normalized to the same prescription dose coverage i.e. D95 $=100 \%$ (95\% of PTV receives at least $100 \%$ of the prescription dose). Two different SRS planning approaches were used to compare $\mathrm{CP}, \mathrm{NCP}, \mathrm{ARC}$ techniques. The first approach evaluated dose to the brainstem and optic chiasm without compromising on PTV coverage (i.e. $\mathrm{D} 95=100 \%)$. This simulates tumor invasion into nearby critical organs where adequate coverage to PTV is prioritized. The second approach followed the guidelines proposed by Timmerman et al. [9], which have now incorporated into AAPM task group (TG) 101 [10] for normal structures. In this case rigid dose constraints were made to the nearby critical structures. This simulates cases where the tumor has no invasion into nearby critical structures and sparing of OARs is highly prioritized.

\subsection{Dosimetric Indices and Their Definitions}

The Eclipse planning system (Varian medical systems, Palo Alto, CA) calculated the following indices after computation of final dose calculation.

Conformity Index (C.I.) evaluates the dose conformity inside the PTV and is calculated as follows: $\mathrm{CI}=\mathrm{V}_{95 \%} / \mathrm{V}_{\mathrm{PTV}}$, where $\mathrm{V}_{95 \%}$ is the volume enclosed by prescription isodose surface (95\%) and $\mathrm{V}_{\mathrm{PTV}}$ is the target volume. C.I. close to 1 is considered as an adequate plan for comparison.

Gradient Measure (G.M.) is the difference between the prescription and half of the prescription isodose spheres. G.M. accounts the spread of $50 \%$ isodose away from PTV and is calculated as follows: G.M. $=R_{50}-R_{p}$, where $R_{p}$ and $R_{50}$ are the equivalent sphere radius of the prescription and half prescription isodoses. A plan that has lowest value of G.M. is considered an adequate plan for comparison.

Mean gradient fall-off calculates the dose fall off in a central (single) slice of PTV in a 2-dimentional area. This measures the difference between the average dose to the $100 \%$ and $50 \%$ two-dimensional isodose surfaces $\left(\mathrm{IS}_{100 \%}\right.$ and $\mathrm{IS}_{50 \%}$ ) in anterior and posterior directions with respect to PTV.

Mean gradient fall-off $=$ Average[(ISant ${ }_{50 \%}-$ ISant $\left._{100 \%}\right)-\left(\right.$ ISpost $_{50 \%}-$ ISpost $\left.\left._{100 \%}\right)\right]$.

A plan that has lowest value of mean gradient fall-off is considered an adequate plan for comparison. 


\section{Results}

Dosimetric indices of standard rapid arc techniques using coplanar (CP) and non-coplanar (NCP) beam arrangements were compared to the proposed cARC technique. Axial (top row) and sagittal (bottom row) isodose distributions are presented for "antPTVs" in Figure 3 and for "postPTVs" in Figure 4 respectively. Sharp dose fall-off in the anterior-posterior direction with respect to the PTV is seen for both "Ant PTVs" and "Post PTVs" using cARC technique (Figure 3 and Figure 4).

Dose volume histograms (DVHs) comparing all three approaches are shown in Figure 5. Comparing cARC with CP, the D50 and D30 of brainstem was reduced by $85.1 \% \pm 3.9 \%$ and $87.6 \% \pm 3.2 \%$ for "Post PTVs", by $51.1 \% \pm 17.8 \%$ and by $85.6 \% \pm 6.0 \%$ for "Ant PTVs" ( $\mathrm{p} \leq 0.01$ ). For chiasm, the reductions were $61.7 \% \pm 3.2 \%$ and $44.2 \% \pm 8.9 \%$ for "Ant PTVs", and $69.3 \% \pm 8.0 \%$ and $74.3 \% \pm$ $8.2 \%$ for "Post PTVs" ( $\mathrm{p} \leq 0.01)$. When comparing cARC with the NCP technique, D50 and D30 of brainstem were reduced by $80.9 \% \pm 3.3 \%$ and $84.6 \% \pm$ $4.8 \%$ for "Post PTVs", by $74.4 \% \pm 4.8 \%$ and $79.6 \% \pm 6.6 \%$ for "Ant PTVs" (p $\leq$ 0.01 ). In addition, for chiasm reductions were $49.9 \% \pm 2.6 \%$ and $34.8 \% \pm 8.2 \%$ for "Ant PTVs" and by $84.8 \% \pm 2.9 \%$ and $86.7 \% \pm 2.5 \%$ for "Post PTVs" ( $\mathrm{p} \leq$ 0.01 ). Profiles of the dose fall-offs for all 3 techniques which were drawn at the center of "Ant PTVs" and anterior to "Post PTVs" are shown in Figure 6.

Comparison of brainstem and chiasm dose for $\mathrm{CP}, \mathrm{NCP}$ and cARC techniques that followed the PTV:D95 $=100 \%$ criteria are shown in Table 2 . As defined by TG101 [10], the maximum point dose where a "point" is defined as $0.035 \mathrm{cc}$ or less, for brainstem and chiasm was limited to $15 \mathrm{~Gy}$ and $10 \mathrm{~Gy}$ respectively. Significant OAR dose reduction was observed for the cARC technique relative to $\mathrm{CP}$ and NCP techniques, particularly to brainstem and chiasm as shown in Figure 5.

With regards to the constraints outlined by Timmerman et al. [9] and now part of TG101 [10] treatment plan quality was assessed by comparing D95 (dose received by $95 \%$ of the volume) and the mean dose to the PTV while satisfying the aforementioned criteria. This allows fulfillment of the study goal of evaluating which technique can better maintain high mean dose and good coverage to PTV after meeting the dose constraints to critical structures. Sparing normal tissues and/or good coverage of the PTV is a foundation of the clinical decision making by radiation oncologist. The cARC technique resulted an overall increase in D95 and mean dose to PTV as compared to CP and NCP as shown in Table 3.

Conformity index (C.I.) analysis is shown in Table 4 demonstrating distinct differences between the CARC technique and standard CP and NCP techniques. The conformity index (C.I., mean $\pm \mathrm{SD}$ ) was measured $1.101 \pm 0.038,1.088 \pm$ 0.054 , and $1.060 \pm 0.040$ for cARC, CP and NCP respectively.

The overall gradient measure (G.M. in $\mathrm{cm}$, mean \pm SD) was $0.581 \pm 0.097$, $0.708 \pm 0.064$, and $0.476 \pm 0.050$ for cARC, CP and NCP respectively. Even 


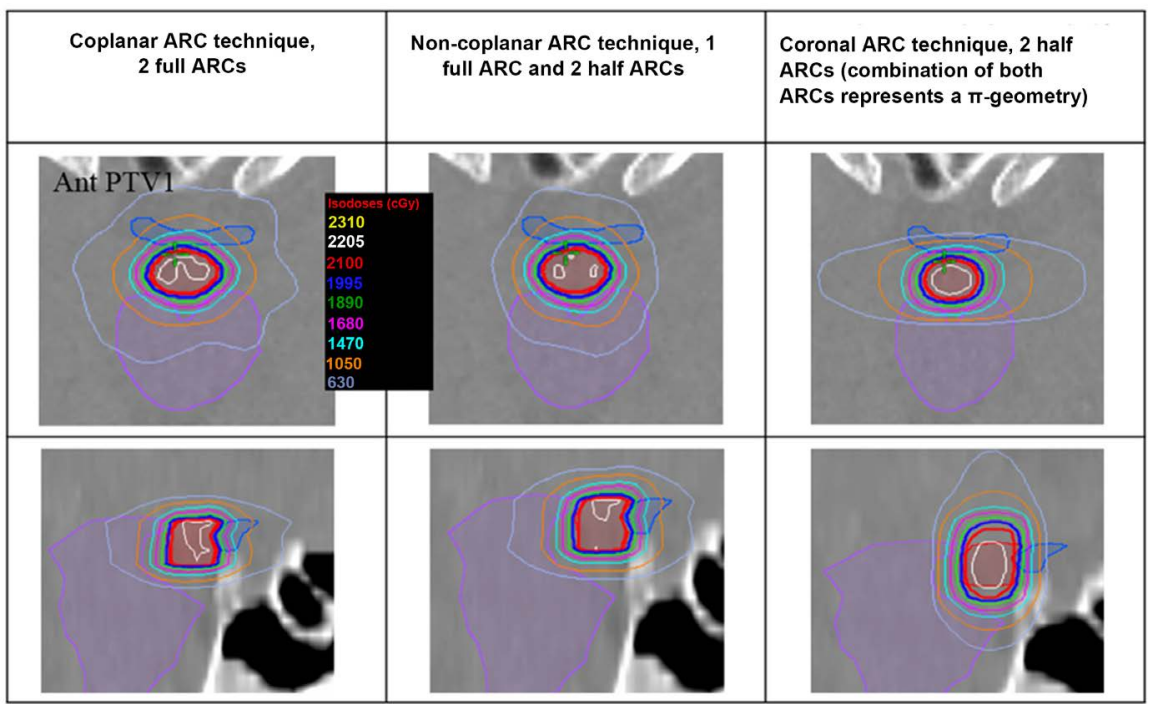

Figure 3. Axial and sagittal isodose distributions for "Ant PTV1" using coplanar (CP), non-coplanar (NCP) and coronal arc (cARC) techniques. By looking at the dose distribution, cARC shows superior dose fall-off in the anterior-posterior direction.

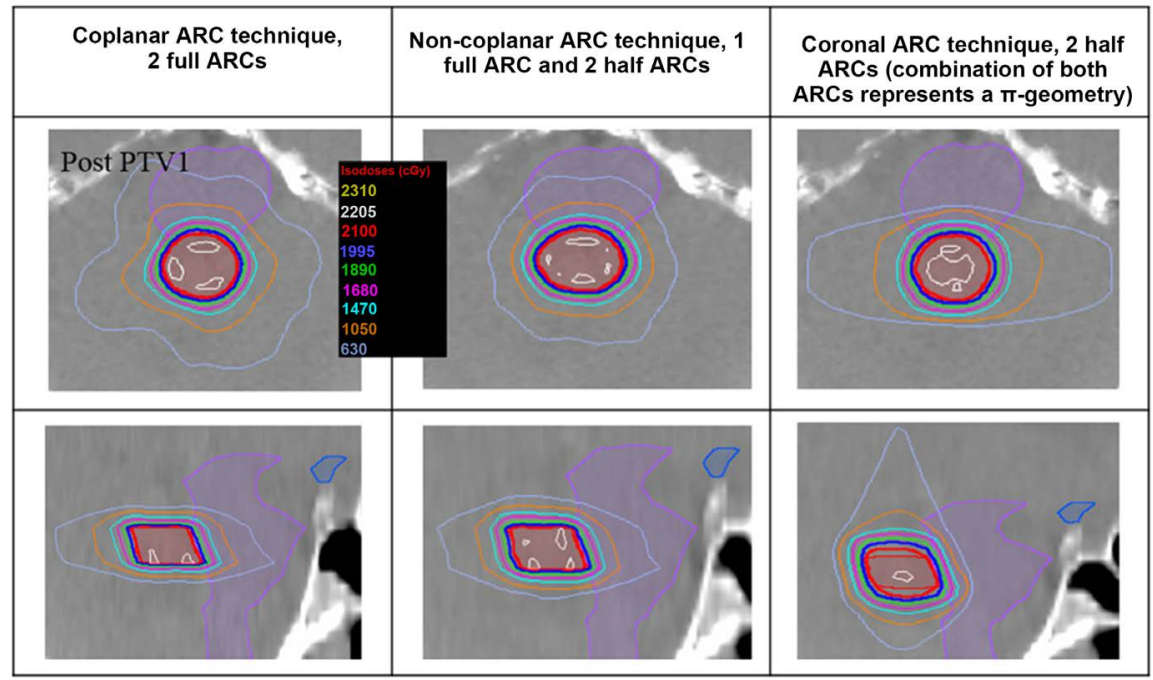

Figure 4. Axial and sagittal isodose distributions for "Post PTV1" using coplanar (CP), non-coplanar (NCP) and coronal arc (cARC) techniques. The cARC technique shows superior dose fall-off in anterior-posterior direction. NCP technique shows compact dose distribution around the PTV as compared to both CP and NCP.

though the gradient measure value of cARC was found inferior to NCP and superior to CP. This parameter is measured spherically. From the dose distribution of cARC, we are mainly focused on saving the OARs anteriorly and posteriorly. Thus, in addition to G.M., a parameter called mean distance gradient fall off was calculated. This provides a clear indication of dose fall off in a plane ( 2 dimensional) rather than 3 dimensional.

The mean distance gradient fall-off (in $\mathrm{cm}$, mean $\pm \mathrm{SD}$ ) was measured as $0.249 \pm 0.038$ (cARC), $0.749 \pm 0.107(\mathrm{CP})$, and $0.621 \pm 0.068(\mathrm{NCP})$ at the center 

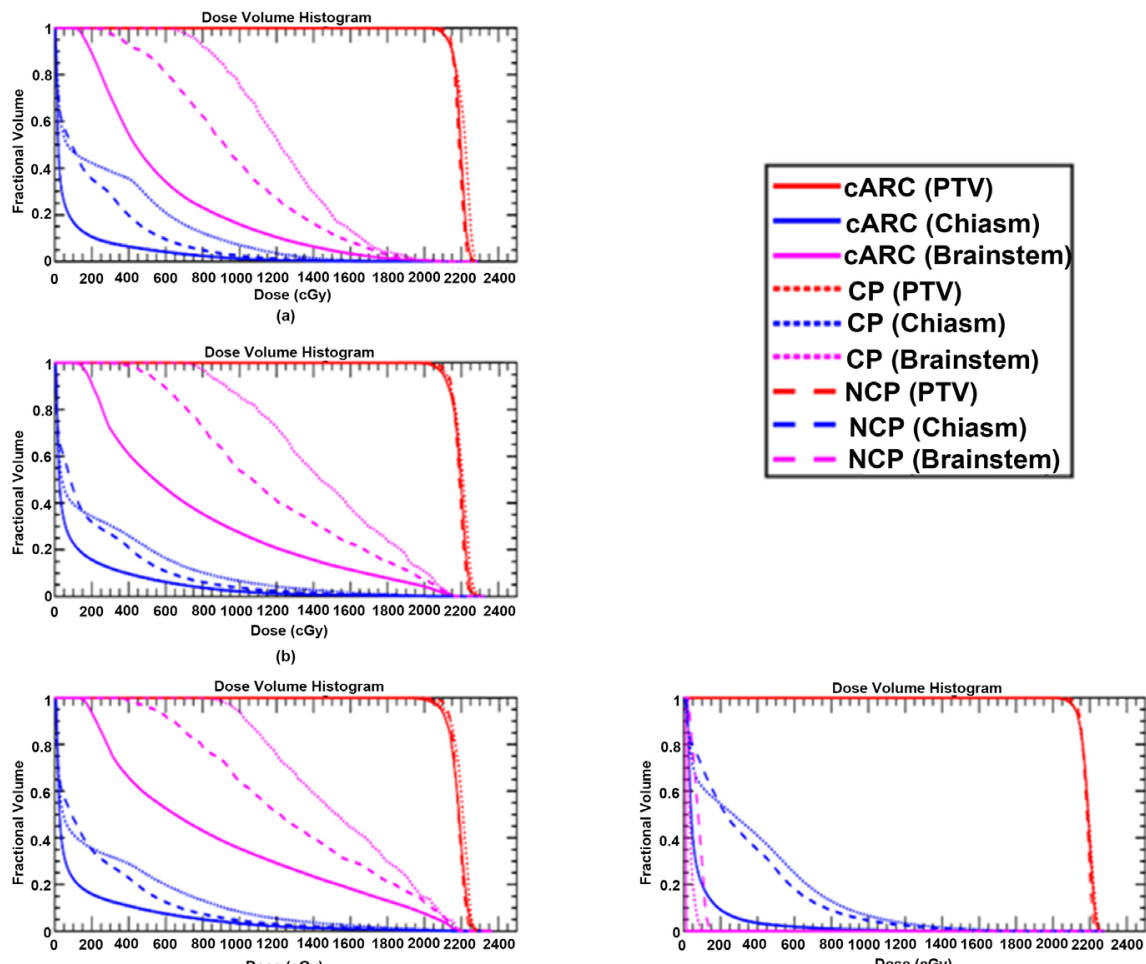

(c)

(f)

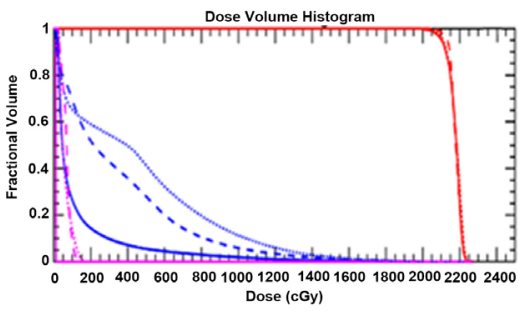

(d)
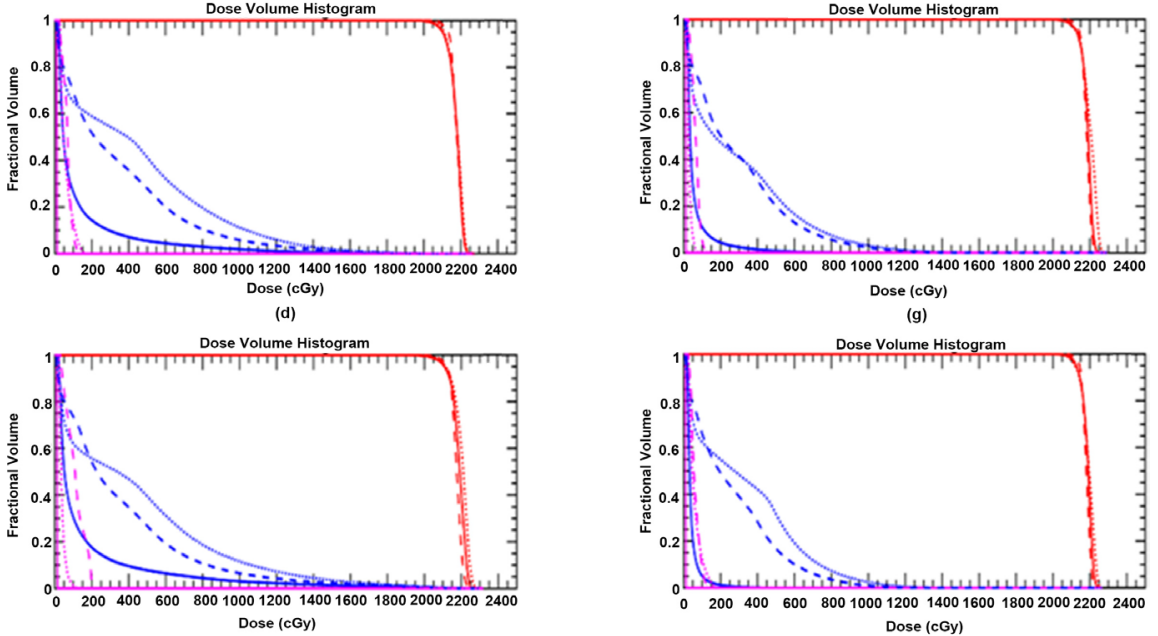
Dose (cGy)
(h)

Figure 5. Comparison of fractional volume vs dose to "Ant PTVs" and "Post PTVs", Chiasm and Brainstem for coplanar (CP), non-coplanar (NCP) and coronal arc (cARC) techniques. Graph "a-c" are for "Ant PTVs" and "d-h" for "Post PTVs". To represent a true comparison, all the plans were normalized equally. It clearly shows an improvement of dose reduction to brainstem and optical chiasm.

slice in anterior-posterior direction of PTV, clearly demonstrating a sharper gradient in the anterior-posterior direction.

\section{Discussion}

This work demonstrates that cARC applied to specific clinical applications can be a significant advancement over existing treatment planning techniques. This 


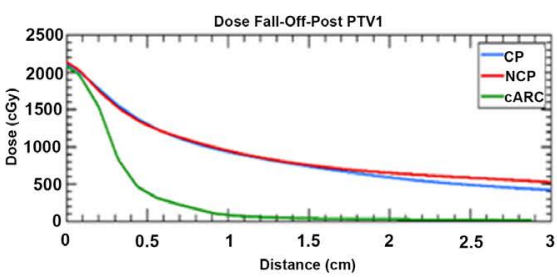

(a)

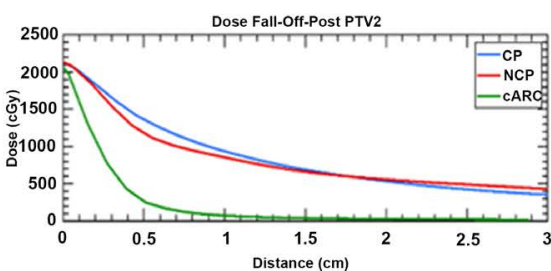

(b)

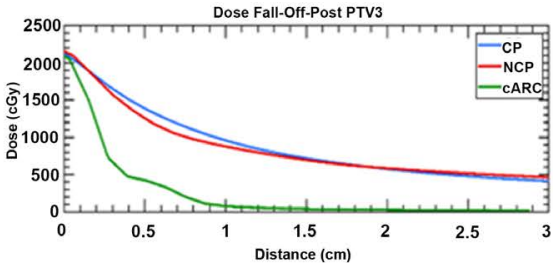

(c)

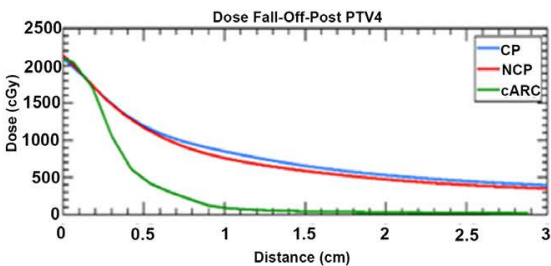

(d)

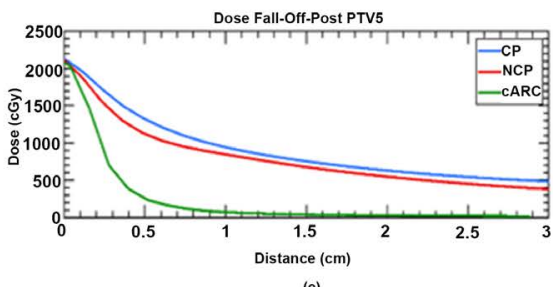

(e)
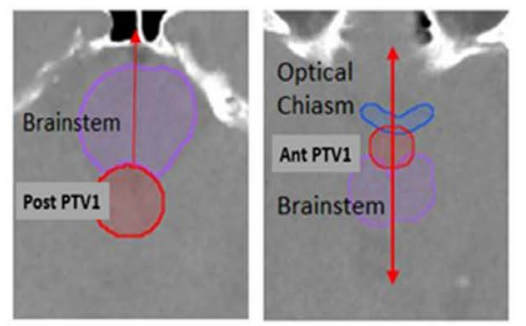

(f)

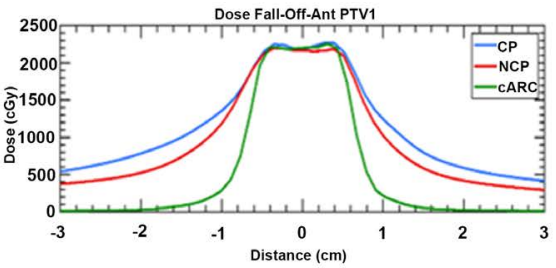

(h)

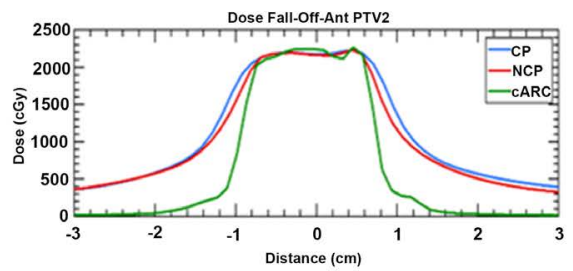

(i)

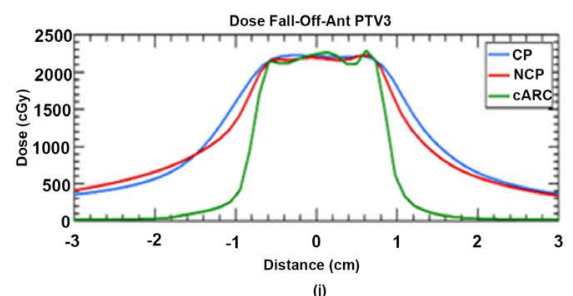

Figure 6. Dose fall-off is shown here which was calculated by drawing a line profile at the center of "Ant PTVs" and anterior to center of "Post PTVs". For example, this figure shows dose fall-off for "Post PTVs" (as shown in graph "a-e") with its profile directionality is shown in graph "(f)" (for e.g. "Post PTV1"). Similar way it is shown for "Ant PTVs" in graph "(h)-(j)" and their directionally (for e.g. "Ant PTV1") in graph "g”).

approach is particularly advantageous in brain when the treatment target is in close proximity to the brainstem and/or optic chiasm. Several investigators have studied the potential of trajectory-based treatment with integration of couch motion during dose delivery [1] [2] [3] [4] [5] [11]-[19]. We have focused on a single- $\pi$ geometry rather than more complex treatment designs which are difficult to deliver and are more likely to result in collision with the patient or gantry. Further, single- $\pi$ geometry has been shown to deliver comparable integral dose to the irradiated volume, sharp dose fall off in the anterior-posterior direction 
Table 2. Comparison of brainstem and chiasm dose for CP, NCP and cARC techniques following PTV:D95 = 100\% criteria. As per TG101, max point dose where "point" is defined as $0.035 \mathrm{cc}$ or less, for brainstem and chiasm it is limited to $15 \mathrm{~Gy}$ and $10 \mathrm{~Gy}$. The V10 Gy to brainstem and V8 Gy to chiasm are the threshold doses. $D_{\leq 0.5 c c}$ for brainstem and $\mathrm{D}_{\leq 0.2 c c}$ for chiasm are chosen as meaningful points for "max critical volume above threshhold".

\begin{tabular}{|c|c|c|c|c|c|c|c|}
\hline \multirow[b]{2}{*}{ PTV } & \multirow{2}{*}{$\begin{array}{l}\text { Planning } \\
\text { Technique }\end{array}$} & \multicolumn{3}{|c|}{ Brainstem } & \multicolumn{3}{|c|}{ Chiasm } \\
\hline & & $\begin{array}{l}\mathrm{D}_{\leq 0.5 \mathrm{cc}} \\
(\mathrm{cGy})\end{array}$ & $\begin{array}{c}V \geq 10 \mathrm{~Gy} \\
(\mathrm{cc})\end{array}$ & $\begin{array}{l}\mathrm{D}_{0.035 \mathrm{cc}} \\
(\mathrm{cGy})^{*}\end{array}$ & $\begin{array}{l}\mathrm{D}_{\leq 0.2 \mathrm{cc}} \\
(\mathrm{cGy})\end{array}$ & $\begin{array}{c}V \geq 8 \text { Gy } \\
(c c)\end{array}$ & $\begin{array}{l}D_{0.035 c c} \\
(c G y)^{*}\end{array}$ \\
\hline \multirow{3}{*}{ Ant PTV1 } & $\mathrm{CP}$ & 1375 & 2.21 & 1811 & 1477 & 0.83 & 1818 \\
\hline & NCP & 1112 & 0.75 & 1731 & 1280 & 0.56 & 1743 \\
\hline & cARC & 917 & 0.36 & 1591 & 809 & 0.21 & 1552 \\
\hline \multirow{3}{*}{ Ant PTV2 } & $\mathrm{CP}$ & 1609 & 2.07 & 2074 & 1827 & 0.87 & 2087 \\
\hline & $\mathrm{NCP}$ & 1362 & 1.16 & 2064 & 1605 & 0.66 & 2073 \\
\hline & cARC & 1095 & 0.66 & 1989 & 1134 & 1133 & 2013 \\
\hline \multirow{3}{*}{ Ant PTV3 } & $\mathrm{CP}$ & 1772 & 2.59 & 2146 & 1917 & 0.89 & 2155 \\
\hline & $\mathrm{NCP}$ & 1514 & 1.44 & 2176 & 1780 & 0.72 & 2128 \\
\hline & cARC & 1307 & 1.03 & 2133 & 1421 & 0.38 & 2104 \\
\hline \multirow{3}{*}{ Post PTV1 } & $\mathrm{CP}$ & 1545 & 3.54 & 1936 & 82 & -- & 132 \\
\hline & NCP & 1462 & 1.85 & 1914 & 80 & -- & 113 \\
\hline & cARC & 1071 & 0.59 & 1752 & 12 & -- & 13 \\
\hline \multirow{3}{*}{ Post PTV2 } & $\mathrm{CP}$ & 1795 & 3.63 & 2154 & 47 & -- & 79 \\
\hline & $\mathrm{NCP}$ & 1666 & 2.03 & 2144 & 45 & -- & 75 \\
\hline & cARC & 1394 & 0.94 & 2113 & 14 & -- & 14 \\
\hline \multirow{3}{*}{ Post PTV3 } & $\mathrm{CP}$ & 1399 & 2.08 & 1762 & 48 & -- & 80 \\
\hline & $\mathrm{NCP}$ & 1309 & 1.46 & 1707 & 47 & -- & 79 \\
\hline & cARC & 656 & 0.14 & 1253 & 12 & -- & 13 \\
\hline \multirow{3}{*}{ Post PTV4 } & $\mathrm{CP}$ & 1138 & 0.98 & 1436 & 36 & -- & 57 \\
\hline & $\mathrm{NCP}$ & 1074 & 0.72 & 1361 & 35 & -- & 55 \\
\hline & cARC & 375 & -- & 738 & 12 & -- & 11 \\
\hline \multirow{3}{*}{ Post PTV5 } & $\mathrm{CP}$ & 1063 & 0.76 & 1288 & 78 & -- & 137 \\
\hline & NCP & 926 & 0.27 & 1151 & 75 & -- & 111 \\
\hline & cARC & 173 & -- & 376 & 11 & -- & 12 \\
\hline
\end{tabular}

*Volume receiving max point dose where "point" is defined as $0.035 \mathrm{cc}$ or less.

and less dose spread to brainstem and optic chiasm relative to highly complex $4 \pi$-geometry treatment plans [11] [12] [13] [14] [17].

Our results demonstrate that the cARC beam arrangement provides better normal tissue sparing and comparable dose coverage to the target as compared to existing planning techniques of CP \& NCP [1] [3] [4] [5] [16] [19]. This is evidenced by the various dose metrics sampled including reduced dose to 
Table 3. Comparison of D95 (cGy) and mean dose to "Ant PTVs" and "Post PTVs" for all techniques satisfying TG101 criteria of dose constraints to brainstem $(0.5 \mathrm{cc}<10 \mathrm{~Gy}$, max point dose $<15 \mathrm{~Gy}$ ) and optic chiasm (0.2 cc $<8 \mathrm{~Gy}$, max point dose $<10 \mathrm{~Gy})$.

\begin{tabular}{|c|c|c|c|}
\hline PTV & Technique & PTV D95 (cGy) & Mean dose to PTV (cGy) \\
\hline \multirow{3}{*}{ Ant PTV1 } & $\mathrm{CP}$ & 1273 & 1523 \\
\hline & NCP & 1508 & 1907 \\
\hline & cARC & 1684 & 2045 \\
\hline \multirow{3}{*}{ Ant PTV2 } & $\mathrm{CP}$ & 1031 & 1335 \\
\hline & NCP & 1068 & 1702 \\
\hline & cARC & 1658 & 2050 \\
\hline \multirow{3}{*}{ Ant PTV3 } & $\mathrm{CP}$ & 978 & 1282 \\
\hline & NCP & 1051 & 1698 \\
\hline & cARC & 1445 & 2070 \\
\hline \multirow{3}{*}{ Post PTV1 } & $\mathrm{CP}$ & 1802 & 2096 \\
\hline & NCP & 2106 & 2176 \\
\hline & cARC & 2120 & 2170 \\
\hline \multirow{3}{*}{ Post PTV2 } & $\mathrm{CP}$ & 1598 & 2045 \\
\hline & NCP & 1638 & 2103 \\
\hline & cARC & 1850 & 2150 \\
\hline \multirow{3}{*}{ Post PTV3 } & $\mathrm{CP}$ & 2082 & 2182 \\
\hline & NCP & 2138 & 2176 \\
\hline & cARC & 2140 & 2178 \\
\hline \multirow{3}{*}{ Post PTV4 } & $\mathrm{CP}$ & 2118 & 2177 \\
\hline & NCP & 2144 & 2178 \\
\hline & cARC & 2145 & 2180 \\
\hline \multirow{3}{*}{ Post PTV5 } & $\mathrm{CP}$ & 2120 & 2179 \\
\hline & NCP & 2145 & 2180 \\
\hline & cARC & 2150 & 2185 \\
\hline
\end{tabular}

normal structures in terms of mean dose and max dose, comparable conformity index, and high dose fall-off obtained using the cARC technique relative to $\mathrm{CP}$ and NCP. Conformity Index (C.I.) for cARC is comparable to CP and NCP techniques. This suggests that the cARC technique can potentially be implemented for intracranial applications.

Further justification of this approach is validated by our results with regards to G.M. and mean gradient fall off. Although G.M. for cARC was reduced relative to $\mathrm{CP}$ and comparable to $\mathrm{NCP}$, the parameter is not necessarily appropriate for our analysis since G.M. is evaluated isotropically without any consideration of laterality. An appropriate analogy to describe the advantage of cARC technique is to compare dose distribution to the deformation of a balloon. Dose 
Table 4. Conformity Index (C.I.) and Gradient Measure (G.M.) comparison of coplanar (CP), non-coplanar (NCP) and coronal arc (cARC) planning techniques. Non-coplanar (NCP) technique has better C.I. and G.M. indices. Conformity Index (C.I.) for cARC is comparable to both NCP and CP. Gradient Measure (G.M.) for cARC resides in between NCP and CP but superior to CP.

\begin{tabular}{ccccccc}
\hline & \multicolumn{3}{c}{ Conformity Index (C.I.) } & \multicolumn{3}{c}{ Gradient Measure (G.M.) } \\
\cline { 2 - 7 } PTVs & $\begin{array}{c}\text { Coplanar } \\
\text { Arcs (CP) }\end{array}$ & $\begin{array}{c}\text { Non-Coplanar } \\
\text { Arcs (NCP) }\end{array}$ & $\begin{array}{c}\text { Coronal } \\
\text { Arc } \\
\text { (cARC) }\end{array}$ & $\begin{array}{c}\text { Coplanar } \\
\text { Arcs (CP) }\end{array}$ & $\begin{array}{c}\text { Non-Coplanar } \\
\text { Arcs (NCP) }\end{array}$ & $\begin{array}{c}\text { Coronal Arc } \\
\text { (cARC) }\end{array}$ \\
\hline Ant PTV1 & 1.21 & 1.15 & 1.16 & 0.73 & 0.42 & 0.44 \\
Ant PTV2 & 1.08 & 1.06 & 1.12 & 0.61 & 0.41 & 0.46 \\
Ant PTV3 & 1.08 & 1.03 & 1.04 & 0.63 & 0.42 & 0.50 \\
Post PTV1 & 1.02 & 1.03 & 1.06 & 0.79 & 0.51 & 0.65 \\
Post PTV2 & 1.08 & 1.03 & 1.09 & 0.78 & 0.51 & 0.66 \\
Post PTV3 & 1.09 & 1.05 & 1.10 & 0.70 & 0.50 & 0.63 \\
Post PTV4 & 1.08 & 1.06 & 1.11 & 0.70 & 0.52 & 0.64 \\
Post PTV5 & 1.06 & 1.07 & 1.13 & 0.72 & 0.52 & 0.67 \\
Mean \pm & $1.088 \pm$ & $1.060 \pm$ & $1.101 \pm$ & $0.708 \pm$ & $0.476 \pm$ & $0.581 \pm$ \\
SD & 0.054 & 0.040 & 0.038 & 0.064 & 0.050 & 0.097 \\
\hline
\end{tabular}

distribution can be likened to a balloon; if squeezed in two dimensions it will deform into the third dimension. For cARC, dose spillage into the lateral and superior-inferior direction into the normal brain is accepted, but the critical clinical advantage gained is dose fall-off in the anterior-posterior direction. This is in recognition that for lesions proximal to the brainstem and optic chiasm the greater concern is with the dose fall-off anteriorly and posteriorly. Based on this, mean dose gradient fall-off is a more meaningful parameter than gradient measure because the later applies spherically and degrades the advantage of cARC as compared to CP and NCP.

This work presents two dosimetric approaches representing alternative treatment objectives. For the first approach of assuring D $95=100 \%$ in our comparison of cARC relative to $\mathrm{CP}$ and NCP, an overall lower dose to the optic chiasm and brainstem results (D50 and D30 are significantly reduced). Unfortunately, $\mathrm{D}_{0.035 \mathrm{cc}}$ is increased relative to TG101 targets of $15 \mathrm{~Gy}$ to brainstem and $10 \mathrm{~Gy}$ to optic chiasm. This is caused by the proximity and assumed invasion of "Ant PTV2", "Ant PTV3", "Post PTV1" and "Post PTV2" into OARs.

For the second approach, dose constraints to the brainstem $(0.5 \mathrm{cc}<10 \mathrm{~Gy}$, maximum point dose $<15 \mathrm{~Gy})$ and chiasm $(0.2 \mathrm{cc}<8 \mathrm{~Gy}$, maximum point dose $<10$ Gy) were met. Comparison of the dose to PTV in terms of D95 and mean dose shows that for acceptable plans cARC provides dosimetric improvement relative to CP and NCP for "Ant PTV1", "Ant PTV2", "Ant PTV3", "Post PTV1", and "Post PTV2". When PTV moves away from OARs such as for "Post PTV3", "Post PTV4", and "Post PTV5" the advantage of cARC becomes small 
and, in terms of dosimetry it is comparable to CP and NCP.

Constraining doses to OARs allowed for study of the usefulness of cARC for PTVs which had invaded OARs or have margins within OARs. To have clinically acceptable plan two options must be provided; either save OARs or to provide coverage to the PTV within the OARs. Clearly, a suitable clinically treatment plan must meet the patient condition which may include spread of disease in to the nearby OARs. The ability to control dose to OARs or provide adequate coverage to PTV is a hallmark of cARC.

Implementation of this technique is particularly useful in instances where the lesion is in close proximity or invading the OARs. This is further compounded by the addition of a PTV margin accounting for set-up error. Maintaining coverage of the PTV while respecting the dose constraints of the critical structures is therefore a crucial component of the clinical decision-making process. However, in the current clinical paradigm, lesions in close proximity to the brainstem or chiasm must compromise PTV coverage to respect the dose constraints of the OARs. The cARC technique introduces an alternative approach by shifting the dose distribution away from the OARs while maintaining PTV coverage not previously attainable using the CP and NCP techniques.

While the proposed cARC technique confers significant benefit over the current clinical alternatives, implementation of this technique is limited by the capabilities of the treatment planning and delivery systems. It is therefore necessary to not only develop the framework for coronal arc optimization planning and translate it such a way that it can be delivered in supine position but to also specify quality assurance measures to ensure safe treatment delivery. One possible approach to achieve this goal is to deliver cARC during treatment couch motion by keeping the gantry static. Further, while the utility of this technique is particularly advantageous in brain, opportunities exist for application in various extracranial sites including breast and extremities. Work is currently underway to recognize these goals.

\section{Conclusion}

In conclusion, we have simulated an innovative cARC technique that provides sharp fall-off of dose anteriorly and posteriorly from PTV in brain while minimizing dose to OARs. We propose the benefits of this technique in treatment of SRS and SRT cases for those patients who are candidates of hypo-fractionation regimen.

\section{Conflicts of Interest}

All authors reported no conflict of interest.

\section{References}

[1] MacDonald, R.L. and Thomas, C.G. (2015) Dynamic Trajectory-Based Couch Motion for Improvement of Radiation Therapy Trajectories in Cranial SRT. Medical 
Physics, 42, 2317-2325. https://doi.org/10.1118/1.4917165

[2] Nguyen, D., et al. (2014) Integral Dose Investigation of Non-Coplanar Treatment Beam Geometries in Radiotherapy. Medical Physics, 41, Article ID: 011905.

[3] Papp, D., Bortfeld, T. and Unkelbach, J. (2015) A Modular Approach to Intensity-Modulated Arc Therapy Optimization with Noncoplanar Trajectories. Physics in Medicine \& Biology, 60, 5179-5198. https://doi.org/10.1088/0031-9155/60/13/5179

[4] Smyth, G., et al. (2016) Non-Coplanar Trajectories to Improve Organ at Risk Sparing in Volumetric Modulated Arc Therapy for Primary Brain Tumors. Radiotherapy and Oncology, 121, 124-131. https://doi.org/10.1016/j.radonc.2016.07.014

[5] Yang, Y., et al. (2011) Choreographing Couch and Collimator in Volumetric Modulated Arc Therapy. International Journal of Radiation Oncology Biology Physics, 80, 1238-1247. https://doi.org/10.1016/j.ijrobp.2010.10.016

[6] Paliwal, B. and Tewatia, D. (2009) Advances in Radiation Therapy Dosimetry. Journal of Medical Physics, 34, 108-116. https://doi.org/10.4103/0971-6203.54842

[7] Gregucci, F., et al. (2018) Linac-Based Radiosurgery or Fractionated Stereotactic Radiotherapy with Flattening Filter-Free Volumetric Modulated Arc Therapy in Elderly Patients: A Mono-Institutional Experience on 110 Brain Metastases. Strahlentherapie und Onkologie, 195, 218-225. https://doi.org/10.1007/s00066-018-1405-0

[8] Roa, D.E., et al. (2012) The Use of RapidArc Volumetric-Modulated Arc Therapy to Deliver Stereotactic Radiosurgery and Stereotactic Body Radiotherapy to Intracranial and Extracranial Targets. Medical Dosimetry, 37, 257-264.

https://doi.org/10.1016/j.meddos.2011.09.005

[9] Timmerman, R.D. (2008) An Overview of Hypofractionation and Introduction to This Issue of Seminars in Radiation Oncology. Seminars in Radiation Oncology, 18, 215-222. https://doi.org/10.1016/j.semradonc.2008.04.001

[10] Benedict, S.H., et al. (2010) Stereotactic Body Radiation Therapy: The Report of AAPM Task Group 101. Medical Physics, 37, 4078-4101. https://doi.org/10.1118/1.3438081

[11] Dong, P., et al. (2013) 4pi Noncoplanar Stereotactic Body Radiation Therapy for Centrally Located or Larger Lung Tumors. International Journal of Radiation Oncology Biology Physics, 86, 407-413. https://doi.org/10.1016/j.ijrobp.2013.02.002

[12] Dong, P., et al. (2013) 4pi Non-Coplanar Liver SBRT: A Novel Delivery Technique. International Journal of Radiation Oncology Biology Physics, 85, 1360-1366. https://doi.org/10.1016/j.ijrobp.2012.09.028

[13] Nguyen, D., et al. (2014) Feasibility of Extreme Dose Escalation for Glioblastoma Multiforme Using 4pi Radiotherapy. Radiation Oncology, 9, 239. https://doi.org/10.1186/s13014-014-0239-x

[14] Rwigema, J.C., et al. (2015) 4pi Noncoplanar Stereotactic Body Radiation Therapy for Head-And-Neck Cancer: Potential to Improve Tumor Control and Late Toxicity. International Journal of Radiation Oncology Biology Physics, 91, 401-409. https://doi.org/10.1016/j.ijrobp.2014.09.043

[15] Seppala, J., et al. (2017) Dosimetric Comparison and Evaluation of 4 Stereotactic Body Radiotherapy Techniques for the Treatment of Prostate Cancer. Technology in Cancer Research \& Treatment, 16, 238-245. https://doi.org/10.1177/1533034616682156

[16] Smyth, G., Bamberm J.C., Evans, P.M. and Bedford, J.L. (2013) Trajectory Optimization for Dynamic Couch Rotation during Volumetric Modulated Arc Radiotherapy. Physics in Medicine \& Biology, 58, 8163-8177. 
https://doi.org/10.1088/0031-9155/58/22/8163

[17] Tran, A., et al. (2017) Predicting Liver SBRT Eligibility and Plan Quality for VMAT and 4pi Plans. Radiation Oncology, 12, 70. https://doi.org/10.1186/s13014-017-0806-Z

[18] Woods, K., et al. (2016) Viability of Non-Coplanar VMAT for Liver SBRT as Compared to Coplanar VMAT and Beam Orientation Optimized 4pi IMRT. Radiation Oncology, 1, 67-75. https://doi.org/10.1016/j.adro.2015.12.004

[19] Yu, V.Y., et al. (2015) The Development and Verification of a Highly Accurate Collision Prediction Model for Automated Noncoplanar Plan Delivery. Medical Physics, 42, 6457-6467. https://doi.org/10.1118/1.4932631 